\title{
Resonances in Reverse Vavilov-Cherenkov Radiation Produced by Electron Beam Passage over Periodic Interface
}

\author{
Gerard Granet, ${ }^{1}$ Petr Melezhik, ${ }^{2}$ Anatoliy Poyedinchuk, ${ }^{2}$ Seil Sautbekov, ${ }^{3}$ \\ Yuriy Sirenko, ${ }^{2,3}$ and Nataliya Yashina ${ }^{2}$ \\ ${ }^{1}$ Universite Blaise Pascal, Avenue des Landais 24, 63177 Clermont-Ferrand, France \\ ${ }^{2}$ A.Ya. Usikov Institute of Radiophysics and Electronics, National Academy of Sciences of Ukraine, 12 Ak. Proskury Street, \\ Kharkov 61085, Ukraine \\ ${ }^{3}$ L.N. Gumilyov Eurasian National University, 2 Mirzoyana Street, Astana 010000, Kazakhstan
}

Correspondence should be addressed to Yuriy Sirenko; yks@bk.ru

Received 12 March 2015; Revised 11 July 2015; Accepted 14 July 2015

Academic Editor: Weiren Zhu

Copyright (c) 2015 Gerard Granet et al. This is an open access article distributed under the Creative Commons Attribution License, which permits unrestricted use, distribution, and reproduction in any medium, provided the original work is properly cited.

Resonances in reverse Vavilov-Cherenkov radiation produced by the charged particles beam passage over periodic boundary of dispersive left-handed medium are found out and studied. Analysis and modeling are performed on the base of rigorous mathematical approaches. For the first time, several physical peculiarities owing to these effects are considered in the conditions of possible resonant scattering of electromagnetic waves.

\section{Introduction}

Vavilov-Cherenkov radiation is appreciated as one of the greatest discoveries of the 20th century. The modern history of Vavilov-Cherenkov radiation (VChR) goes back to Cherenkov's experiments, 1934-1937 [1]. Tamm and Frank developed this radiation theory in 1937 [2]. In 1958, the work was awarded a Nobel Prize in physics. Since then, VChR has been extensively used in physics and engineering [3]. As materials science and engineering advance, actual lefthanded media providing qualitatively new characteristics in the implementation of well-known phenomena come and cause a fresh interest in VChR from both theoretical and applied standpoints.

The study presented in the paper considers several still poorly understood peculiarities of the diffraction radiation [4] and, in particular, the direct and reverse VChR [4-7] produced by a beam of charged particles moving above the dispersive medium surface (generally periodic). Depending on frequency, this medium can be right-handed (conventional dielectric), mononegative (either permittivity or permeability is negative), or left-handed (both permittivity and permeability are negative). Emphasis is placed on the radiation characteristics in the conditions of possible resonant scattering of electromagnetic waves when the modulation period of the electron beam is comparable with the interface period and the excitation of eigenwaves supported by this boundary is possible. The study of resonant situations requires adequate research methods and tools that are based mainly on the idea of analytic regularization of boundary value problems in electromagnetic theory of gratings $[8,9]$.

\section{Models and Characteristics}

In free space $(\varepsilon(g)=\mu(g) \equiv 1)$, the eigenfield $\left\{\vec{E}^{i}(g, k), \vec{H}^{i}(g, k)\right\}$ of electron beam with modulated density and the instantaneous value of the charge density given by the expression $\rho \delta(z-a) \exp [i((k / \beta) y-k t)]$ represents the $H$-polarized field $\left(\partial / \partial x \equiv 0, E_{x}^{i}=H_{y}^{i}=H_{z}^{i}=0\right)$ and accordingly [4]

$$
\begin{aligned}
& H_{x}^{i}(g, k)=2 \pi \rho \beta \\
& \cdot \exp \left\{i\left[\sqrt{k^{2}-\left(\frac{k}{\beta}\right)^{2}}|z-a|+\left(\frac{k}{\beta}\right) y\right]\right\}
\end{aligned}
$$




$$
\begin{gathered}
\cdot\left[\frac{|z-a|}{(z-a)}\right] ; \quad z \neq a \\
E_{y}^{i}(g, k)=-\left(\frac{\eta_{0}}{i k}\right) \frac{\partial H_{x}^{i}(g, k)}{\partial z}, \\
E_{z}^{i}(g, k)=\left(\frac{\eta_{0}}{i k}\right) \frac{\partial H_{x}^{i}(g, k)}{\partial y} .
\end{gathered}
$$

Here $\delta(\cdots)$ is Dirac's $\delta$-function; $\rho$ and $k$ are the amplitude and the modulation frequency of the electron beam and $0<\beta<1$ is beam's relative velocity; $\eta_{0}=\left(\mu_{0} / \varepsilon_{0}\right)^{1 / 2}$ is the impedance of free space; $\varepsilon_{0}$ and $\mu_{0}$ are electric and magnetic constants of the vacuum; $\varepsilon(g)$ and $\mu(g)$ are relative permittivity and permeability of the medium in the domain of wave propagation; $g=\{y, z\}$ is a point in the space $R^{2}$; time dependence of the harmonically oscillating fields is determined by the factor $\exp (-i k t)$. All physical quantities have the dimension of the SI, only "time" is measured in meters; it is the product of the real time on the speed of light in vacuum.

$$
\text { Suppose }-2 \pi \rho \beta \sqrt{l} \exp \left[-k(a-h) \sqrt{(1 / \beta)^{2}-1}\right]=1 \text {. }
$$

Then, eigenfield (1) of the electron beam passing in the conventional medium (vacuum) over the periodic boundary $S$ (see Figure 1) of the dispersive medium with constitutive parameters defined by relations

$$
\begin{aligned}
& \varepsilon(k)=1-\frac{k_{\varepsilon}^{2}}{k^{2}}, \\
& \mu(k)=1-\frac{k_{\mu}^{2}}{k^{2}}
\end{aligned}
$$

generates in the domains $z \geq h$ and $z \leq 0$ the $H$ polarized field $\left\{\vec{E}^{s}(g, k), \vec{H}^{s}(g, k)\right\}$ with nonzero components represented as follows [8]:

$$
\begin{aligned}
& H_{x}^{s}(g, k) \\
& =\sum_{n=-\infty}^{\infty} \mu_{n}(y) \begin{cases}R_{n}(k) \exp \left[i \Gamma_{n}(z-h)\right] ; & z \geq h \\
T_{n}(k) \exp \left[-i \Gamma_{n}^{\varepsilon, \mu} z\right] ; \quad z \leq 0,\end{cases} \\
& E_{y}^{s}(g, k)=-\frac{\eta_{0}}{i k \varepsilon(g)} \frac{\partial H_{x}^{s}(g, k)}{\partial z}=\frac{\eta_{0}}{k} \sum_{n=-\infty}^{\infty} \mu_{n}(y) \\
& \quad\left\{\begin{array}{l}
-R_{n}(k) \Gamma_{n} \exp \left[i \Gamma_{n}(z-h)\right] ; \quad z \geq h \\
T_{n}(k) \Gamma_{n}^{\varepsilon, \mu} \varepsilon^{-1}(k) \exp \left[-i \Gamma_{n}^{\varepsilon, \mu} z\right] ; \quad z \leq 0,
\end{array}\right. \\
& E_{z}^{s}(g, k)=\frac{\eta_{0}}{i k^{\varepsilon}(g)} \frac{\partial H_{x}^{s}(g, k)}{\partial y}=\frac{\eta_{0}}{k} \sum_{n=-\infty}^{\infty} \Phi_{n} \mu_{n}(y) \\
& \quad\left\{\begin{array}{l}
R_{n}(k) \exp \left[i \Gamma_{n}(z-h)\right] ; \quad z \geq h \\
T_{n}(k) \varepsilon^{-1}(k) \exp \left[-i \Gamma_{n}^{\varepsilon, \mu} z\right] ; \quad z \leq 0 .
\end{array}\right.
\end{aligned}
$$

Here, $l$ and $0 \leq h<a$ are the period length and height of the corrugation mounts $S=\{g: z=f(y), 0 \leq f(y) \leq h\}$;

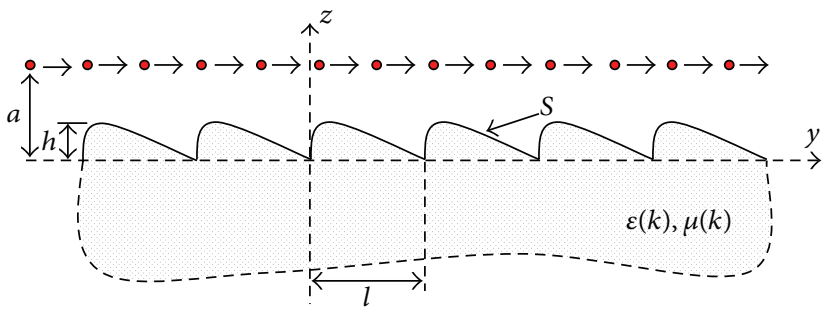

FIGURE 1: Geometry of the problem of diffraction radiation.

$k=2 \pi / \lambda$ and $\lambda$ is the length of the electromagnetic waves in free space; $k_{\varepsilon}$ and $k_{\mu}$ are the characteristic frequencies of dispersive medium; $\mu_{n}(y)=l^{-1 / 2} \exp \left(i \Phi_{n} y\right), \Phi_{n}=2 \pi n / l+\Phi_{0}$, and $\Phi_{0}=k / \beta ; \Gamma_{n}=\sqrt{k^{2}-\Phi_{n}^{2}}$ and $\operatorname{Re} \Gamma_{n} \geq 0, \operatorname{Im} \Gamma_{n} \geq$ 0 . Signs of real and imaginary parts of the $\operatorname{root} \Gamma_{n}^{\mathcal{E}, \mu}=$ $\sqrt{k^{2} \varepsilon(k) \mu(k)-\Phi_{n}^{2}}$ are given so that all partial components $U_{n}^{T}(g, k)=T_{n}(k) \exp \left[-i \Gamma_{n}^{\varepsilon, \mu} z\right] \mu_{n}(y)$ (spatial harmonics) of the field $\left\{\vec{E}^{s}(g, k), \vec{H}^{s}(g, k)\right\}$ in the domain $z \leq 0$ are the outgoing plane waves, that is, homogeneous waves $\left(\operatorname{Im} \Gamma_{n}^{\mathcal{E}, \mu}=\right.$ 0 ), transferring energy in the direction towards $z=-\infty$ or the inhomogeneous waves $\left(\operatorname{Re} \Gamma_{n}^{\mathcal{E}, \mu}=0\right)$, exponentially decaying when moving in the same direction.

Amplitude coefficients $R_{n}(k)$ and $T_{n}(k)$ are complicated function of frequency and geometric and constitutive parameters of the media of wave propagation. We find them by solving numerically the following boundary value problem [8] in the strip $0 \leq y \leq l$ :

$$
\begin{aligned}
& {\left[\frac{\partial^{2}}{\partial y^{2}}+\frac{\partial^{2}}{\partial z^{2}}+k^{2} \varepsilon(g) \mu(g)\right] H_{x}(g, k)=0 ; \quad 0 \leq z \leq h,} \\
& H_{x}\left\{\frac{\partial H_{x}}{\partial y}\right\}(l, z, k)=\exp \left(i \Phi_{0} l\right) H_{x}\left\{\frac{\partial H_{x}}{\partial y}\right\}(0, z, k) ;
\end{aligned}
$$$$
0 \leq z \leq h \text {, }
$$

$$
\begin{aligned}
& H_{x}(g, k)=\sum_{n=-\infty}^{\infty} \mu_{n}(y) \\
& \qquad \begin{cases}\delta_{0}^{n} \exp \left[-i \Gamma_{n}(z-h)\right]+R_{n}(k) \exp \left[i \Gamma_{n}(z-h)\right] ; & z \geq h \\
T_{n}(k) \exp \left[-i \Gamma_{n}^{\varepsilon, \mu} z\right] ; & z \leq 0,\end{cases} \\
& H_{x}(g, k), E_{t g}(g, t)
\end{aligned}
$$

are continuous across $S$ and virtual boundaries $z=0, z$

$$
=h \text {. }
$$

Here, $\delta_{m}^{n}$ is the Kronecker symbol and $H_{x}(g, k)$ is one of the three nonzero components of the total field

$$
\begin{aligned}
& \{\vec{E}(g, k), \vec{H}(g, k)\} \\
& = \begin{cases}\left\{\vec{E}^{i}(g, k), \vec{H}^{i}(g, k)\right\}+\left\{\vec{E}^{s}(g, k), \vec{H}^{s}(g, k)\right\} ; & z \geq h \\
\left\{\vec{E}^{s}(g, k), \vec{H}^{s}(g, k)\right\} ; & z<h .\end{cases}
\end{aligned}
$$

This problem allows determining (within the approximation of a given current) the electromagnetic field (field 
of diffraction radiation) generated by density modulated electron beam $[4,8]$. The method, usually called as a method of analytic regularization $[8,9]$, was used for numerical solving of the problem of interest. The application of this method provided already the majority of the physical and applied results of the electromagnetic theory of gratings associated with resonant and anomalous spatial-frequency and spatial-time transformations of electromagnetic fields in periodic structures $[8,10-13]$.

The energy characteristics of diffraction radiation are given by the relation [8]

$$
\begin{aligned}
& \sum_{n=-\infty}^{\infty}\left[\left|R_{n}\right|^{2} \operatorname{Re} \Gamma_{n}+\left|T_{n}\right|^{2} \operatorname{Re} \Gamma_{n}^{\varepsilon, \mu} \varepsilon^{-1}(k)\right] \\
& \quad=2 \operatorname{Im} R_{0} \operatorname{Im} \Gamma_{0},
\end{aligned}
$$

obtained by the application of the Poynting theorem for complex power to the field $\{\vec{E}(g, k), \vec{H}(g, k)\}$ within the domain $[0 \leq y \leq l] \times[0 \leq z \leq h]$. The term in the left part in (6) is the total electromagnetic energy $W=W^{\uparrow}+W^{\downarrow}$ radiated into the half-spaces $z \geq h$ and $z \leq 0$. In the approximation of the given current, it is determined by the expression $2 \operatorname{Im} R_{0} \operatorname{Im} \Gamma_{0}$. The values $W_{n}^{R}=\left|R_{n}\right|^{2} \operatorname{Re} \Gamma_{n}$ and $W_{n}^{T}=\left|T_{n}\right|^{2} \operatorname{Re} \Gamma_{n}^{\mathcal{\varepsilon}, \mu} \varepsilon^{-1}(k)$ characterize the distribution of the energy, lost by the electrons beam, between the channels open for radiation, that is, between the harmonics of the spatial spectrum such that $\operatorname{Re} \Gamma_{n} \geq$ 0 and/or $\operatorname{Re} \Gamma_{n}^{\varepsilon, \mu} \varepsilon^{-1}(k)>0$. The last inequality and the relationship $\operatorname{Re} P_{y}(k)=\varepsilon^{-1}(k) \sum_{n: \operatorname{Im} \Gamma_{n}^{\varepsilon, \mu}=0}\left|T_{n}\right|^{2} \Phi_{n}\left(P_{y}(k)\right.$ is the $y$-component of the Poynting vector $\vec{P}(k)$ for field $\{\vec{E}(g, k), \vec{H}(g, k)\}$ in the plane $z=0$ averaged over the period $l)$ allow determining unambiguously and quite rigorously the direction of the phase velocity of propagating in the halfspace $z>0$ harmonic $U_{n}^{T}(g, k)$ and the direction in which this harmonic carries the energy. In conventional media, these directions coincide and are given by the vector $\Phi_{n} \vec{y}-\Gamma_{n}^{\varepsilon, \mu} \vec{z}$, $\Gamma_{n}^{\mathcal{E}, \mu}>0$. In binegative media $\Gamma_{n}^{\mathcal{E}, \mu}<0$, the phase velocity is oriented along the vector $\Phi_{n} \vec{y}-\Gamma_{n}^{\mathcal{E}, \mu} \vec{z}$, and the energy transfer holds along the vector $-\Phi_{n} \vec{y}+\Gamma_{n}^{\mathcal{E}, \mu} \vec{z}$. In media with only one negative constitutive parameter the harmonics $U_{n}^{T}(g, k)$ carrying the energy in the direction $z=-\infty$ are forbidden and cannot be excited.

For $\left\{\vec{E}^{i}(g, k), \vec{H}^{i}(g, k)\right\} \equiv 0$ and $k>0$ fixed, the homogeneous (spectral) problem is obtained from (4a)-(4c). Its nontrivial solutions $H_{x}\left(g, \bar{\Phi}_{0(m)}\right)$ exist for no more than a countable set of eigenvalues $\left\{\bar{\Phi}_{0(m)}\right\}_{m} \in F$ and define the field of eigenwaves $\left\{\vec{E}\left(g, \bar{\Phi}_{0(m)}\right), \vec{H}\left(g, \bar{\Phi}_{0(m)}\right)\right\}$ of the periodic interface $[8,10]$. If several eigenvalues belong to the real axis $\operatorname{Re} \Phi_{0}$ of the first physical sheet of the surface $F$ (i.e., a Riemann surface onto which the solution of the problem (4a)-(4c) can be analytically continued from the domain of real values of the spectral parameter $\Phi_{0}$ ), then we are dealing with conventional surface (or regular) waves propagating near the interface without attenuation. Otherwise, leaky waves, piston-type waves, and so forth do exist [8].

\section{Physical Results: Plane Boundary}

A flat boundary separating a conventional environment (vacuum) and a nonabsorbing dispersion medium (the medium sort discussed below) is able to support direct (or forward) surface waves in the frequency range [10]

$$
K_{2}=\frac{k_{\varepsilon} k_{\mu}}{\sqrt{k_{\varepsilon}^{2}+k_{\mu}^{2}}}<k<\frac{k_{\varepsilon}}{\sqrt{2}}=K_{1} ; \quad k_{\varepsilon}>k_{\mu} .
$$

These waves correspond to the propagation constants $\bar{\Phi}_{0}^{\text {direct }}=k k_{\varepsilon}^{-1} \sqrt{\left(k_{\varepsilon}^{2}-k^{2}\right)\left(k_{\varepsilon}^{2}-k_{\mu}^{2}\right)\left(k_{\varepsilon}^{2}-2 k^{2}\right)^{-1}}$ and velocity $\beta=\beta^{\text {direct }}=k / \bar{\Phi}_{0}^{\text {direct }}$ of synchronously moving electron beam.

Within the range

$$
\frac{k_{\varepsilon}}{\sqrt{2}}<k<\frac{k_{\varepsilon} k_{\mu}}{\sqrt{k_{\varepsilon}^{2}+k_{\mu}^{2}}} ; \quad k_{\varepsilon}<k_{\mu}
$$

this boundary can support backward waves having phase and group velocities oppositely directed and characterized by anomalous dispersion. For such waves, the velocity of the synchronously moving beam is $\beta=\beta^{\text {back }}=k / \bar{\Phi}_{0}^{\text {back }}$ and $\bar{\Phi}_{0}^{\text {back }}=k k_{\varepsilon}^{-1} \sqrt{\left(k_{\varepsilon}^{2}-k^{2}\right)\left(k_{\mu}^{2}-k_{\varepsilon}^{2}\right)\left(2 k^{2}-k_{\varepsilon}^{2}\right)^{-1}}$.

Figure 2 shows $\varepsilon(k)$ and $\mu(k)$, propagation constants of eigensurface waves and velocity of electron beam synchronized with these waves versus frequency $k$. All these dependencies are presented within the ranges defined by (7) and (8). For $k_{\mu}=0.5, k_{\varepsilon}=1.0$, and $0.4472<k<0.7071$, we have the forward waves (Figure 2(b)). For $k_{\mu}=1.0, k_{\varepsilon}=0.5$, and $0.3536<k<0.4472$, the backward waves (Figure 2(a)).

Principal differences in the behavior of these characteristics for forward and backward surface waves are rather obvious. In the frequency range $K_{1}<k<K_{2}$ where the backward waves exist, the medium is binegative: $\varepsilon(k)<0$ and $\mu(k)<0$. At the same time in the domain of the forward waves existence, the binegative medium transforms into a medium with $\varepsilon(k)<0$ and $\mu(k)>0$. Naturally, these differences also affect the characteristics of the field excited by the electron beam.

The VChR into the lower half-space filled with the dispersive medium is possible under the condition $\beta^{2}>$ $[\varepsilon(k) \mu(k)]^{-1}$, which can be satisfied only for the bipositive (conventional medium, direct VChR) or binegative (reverse VChR) media. The dispersion law given by (2) yields the following frequency restriction:

$$
k<K_{0}=\frac{\sqrt{2} k_{\varepsilon} k_{\mu}}{\sqrt{k_{\varepsilon}^{2}+k_{\mu}^{2}+\sqrt{\left(k_{\varepsilon}^{2}-k_{\mu}^{2}\right)^{2}+4 k_{\varepsilon}^{2} k_{\mu}^{2} / \beta^{2}}}} .
$$

It can be shown that the inequality $K_{0}<k_{\varepsilon} k_{\mu} / \sqrt{k_{\varepsilon}^{2}+k_{\mu}^{2}}$ always holds if $0<\beta<1$. And as $\beta \rightarrow 0$, then $K_{0} \rightarrow 0$. As $\beta \rightarrow 1, K_{0} \rightarrow k_{\varepsilon} k_{\mu} / \sqrt{k_{\varepsilon}^{2}+k_{\mu}^{2}}$. In Figure 2, the VChR domain is darkened. 


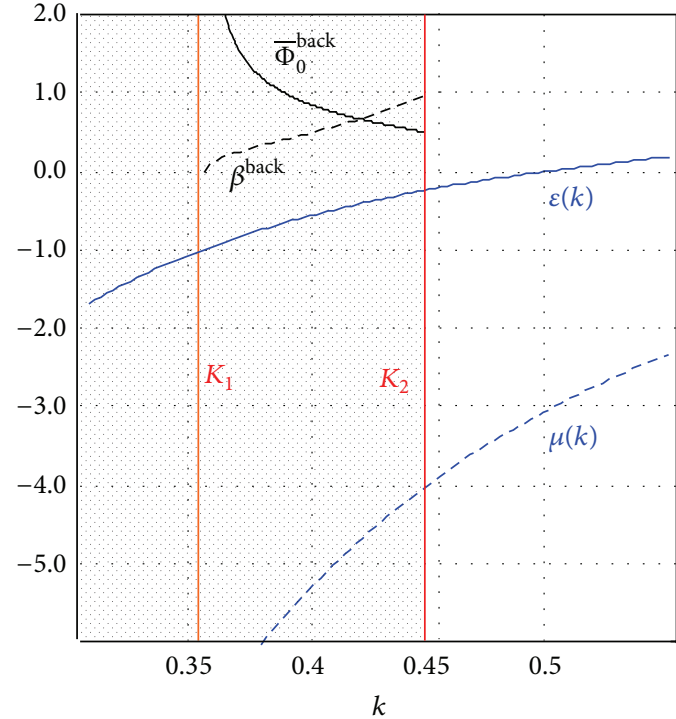

(a)

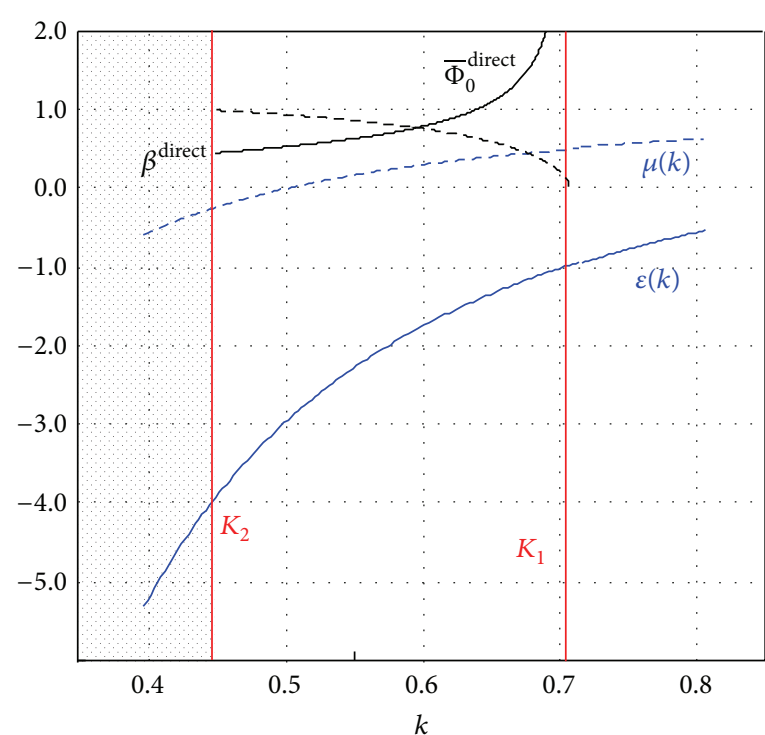

(b)

FIGURE 2: Curves of $\varepsilon(k), \mu(k), \bar{\Phi}_{0}^{\text {back }}, \bar{\Phi}_{0}^{\text {direct }}, \beta^{\text {back }}$, and $\beta^{\text {direct }}$.

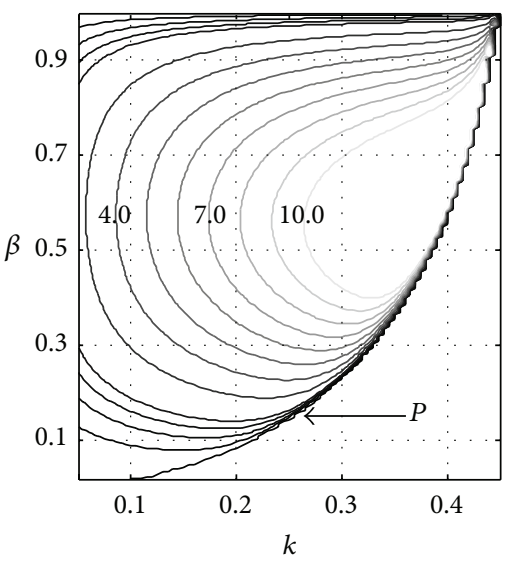

(a)

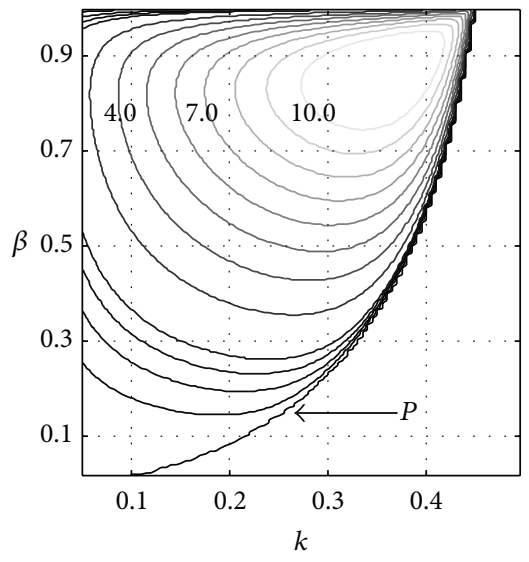

(b)

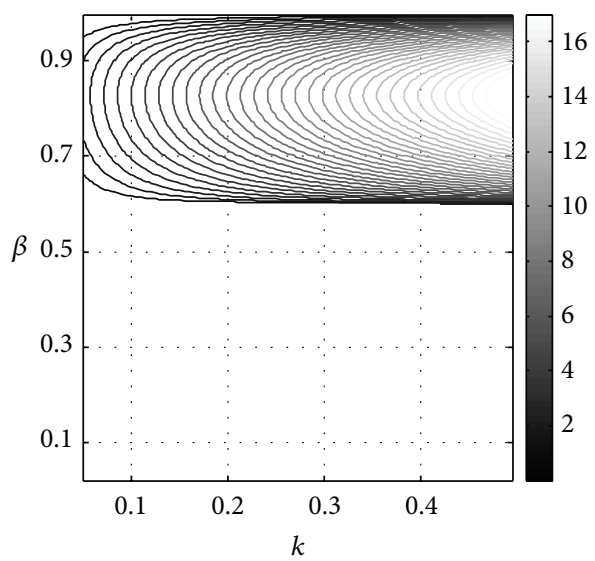

(c)

FIGURE 3: Contour plots of $W_{0}^{T}(k, \beta)=$ const, characterizing the radiation intensity in the half-space $z<0$ : (a) $k_{\varepsilon}=0.5$ and $k_{\mu}=1.0$; (b) $k_{\varepsilon}=1.0$ and $k_{\mu}=0.5$; (c) $\varepsilon=-2.24, \mu=-1.24$, and $\beta=(\varepsilon \mu)^{-1 / 2} \approx 0.6$.

In the case $k_{\varepsilon}<k_{\mu}$, the frequency region of the VChR and the backward surface wave region overlap. On the contrary, when $k_{\varepsilon}>k_{\mu}$, the domain of the forward surface waves is beyond the domain of possible observation of the VChR.

At $h=0$, only zeroth spatial harmonics with amplitudes $R_{0}(k)$ and $T_{0}(k)$ remain in (4a)-(4c). The zeroth spatial harmonic in the dispersive material becomes propagating, that is, transferring the energy infinitely far in the direction $z=-\infty$ provided $\operatorname{Im} \Gamma_{n}^{\mathcal{E}, \mu}(k)=0$, or what is the same, provided $\beta^{2}>[\varepsilon(k) \mu(k)]^{-1}$, which is necessary for the VChR existence. Both the diffraction radiation and the VChR are associated with the transfer of electromagnetic energy in the same direction. Therefore, following [4], we identify the Vavilov-Cherenkov radiation with the diffraction radiation on the fundamental (zeroth) spatial harmonic of the periodic interface.

Figure 3 shows the contour plots $W_{0}^{T}(k, \beta)=$ const, describing the radiation intensity of $\mathrm{VChR}$ in the half-space $z<0$. Various fragments correspond to the media with different parameters $\varepsilon(k)$ and $\mu(k)$. And everywhere, as one would expect, the range of values $k$ and $\beta$ in which $W_{0}^{T}(k, \beta)>$ 0 is bounded by curves $P: \beta=[\varepsilon(k) \mu(k)]^{-1 / 2}$ restricting the domain of VChR existence.

In the case $k_{\varepsilon}>k_{\mu}$ (Figure 3(b)), a maximum VChR intensity region is shifted towards higher $\beta(0.75<\beta<$ $0.95)$ and it is concentrated in the vicinity of the curve $P$. In the case $k_{\varepsilon}<k_{\mu}$ (Figure 3(a)), a similar area is again located near $P$, but shifted towards $0.4<\beta<0.75$. These 


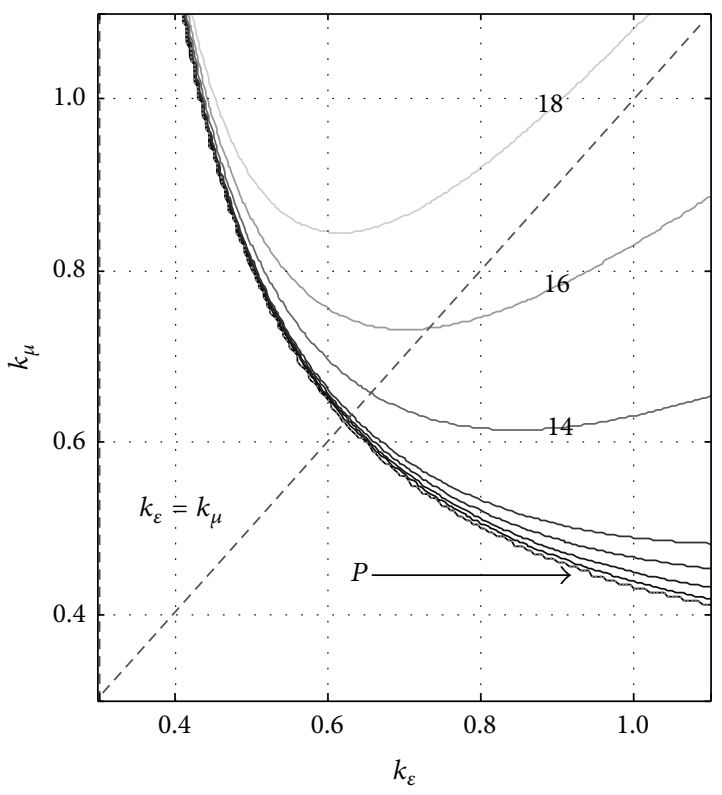

(a)

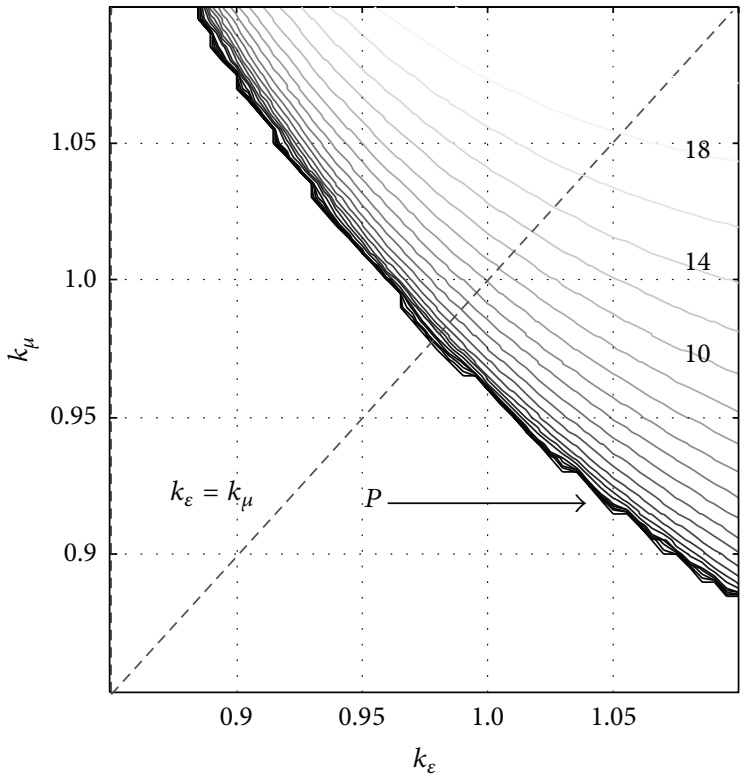

(b)

FIgURE 4: Contour plots for $W_{0}^{T}\left(k_{\varepsilon}, k_{\mu}\right)=$ const: (a) $k=0.3$ and $\beta=0.3$; (b) $k=0.6$ and $\beta=0.6$.

behavioral features of energy characteristics are observed only in the dispersive medium. For a dispersion-free medium (Figure 3(c)) $\max _{(\varepsilon \mu)^{-1 / 2}<\beta<1} W_{0}^{T}(k, \beta)$ is attained at any $k$, but $\beta \approx 0.83$ keeps unchanged. The value $\max _{(\varepsilon \mu)^{-1 / 2}<\beta<1} W_{0}^{T}(k, \beta)$ grows with $k$.

The contour plots $W_{0}^{T}\left(k_{\varepsilon}, k_{\mu}\right)=$ const in Figure 4 give a fairly complete picture of the influence of the constitutive parameters of the dispersive medium with a plane boundary on the VChR energy characteristics. Interestingly, in the case of large $k$ and $\beta$ (in Figure $4(\mathrm{~b})$ ), the lines $W_{0}^{T}\left(k_{\varepsilon}, k_{\mu}\right)=$ const intersect with straight line $k_{\varepsilon}=k_{\mu}$ (the most rapid variation of VChR intensity) at an almost right angle, while for smaller $k$ and $\beta$ (see Figure $4(\mathrm{a})$ ) and $k_{\varepsilon}>0.8$ the passage along the line $k_{\varepsilon}=k_{\mu}$ does not lead to a noticeable change in the VChR intensity.

\section{Physical Results: Periodically Rough Boundary}

In the case of a periodically rough boundary $S$ (further on we assume $l=2 \pi)$, the radiation field in the domain $z \geq h(z<$ $0)$ consists of spatial harmonics $U_{n}^{R}(g, k)=R_{n}(k) \exp \left[i \Gamma_{n}(z-\right.$ $h)] \mu_{n}(y)\left(U_{n}^{T}(g, k)\right)$ propagating without attenuation. So, it is represented by harmonics with such $n$ that $\operatorname{Im} \Gamma_{n}=0$ $\left(\operatorname{Im} \Gamma_{n}^{\mathcal{E}, \mu}=0\right)$. Obviously, when $\Phi_{0}=k / \beta$ only harmonics with negative $n$ can propagate without attenuation in the domain $z \geq h$. In a dispersive medium, the range of $k$ and $\beta$ providing the propagation without decay of one or another spatial harmonic $U_{n}^{T}(g, k)$ can be easily determined using the data on the configuration of their limits $Q_{n}: \Gamma_{n}^{\mathcal{E}, \mu}(k, \beta)=0$ (see, e.g., Figure 5(a)).
A periodically rough boundary supports a larger number of surface waves. Their propagation constants in the frequency range of interest $(k<1)$ and small $h$ are given by the approximate relations $\bar{\Phi}_{0}^{\text {direct, }, m}= \pm \bar{\Phi}_{0}^{\text {direct }}+m$ and $\bar{\Phi}_{0}^{\text {back, }, m}= \pm \bar{\Phi}_{0}^{\text {back }}+m, m=0, \pm 1, \pm 2, \ldots[8$, 10]. Accordingly, we define the sets of approximate values $\beta^{\text {direct, }, m}=k / \bar{\Phi}_{0}^{\text {direct }, \pm, m}$ and $\beta^{\text {back }, \pm, m}=k / \bar{\Phi}_{0}^{\text {back }, \pm, m}$ (see Figures $5(\mathrm{~b})$ and $5(\mathrm{c})$ ). It should be pointed out that all the curves corresponding to velocities $\beta^{\text {direct, } \pm, m}$ and $\beta^{\text {back, }, m}$ practically merge near the values of the frequency parameter $k=k_{\text {accum }}=k_{\varepsilon} / \sqrt{2}$, which for the given dispersion law (2) define accumulation point of the frequency spectra of the electrodynamic structure [13]. At this point, $\varepsilon(k)=-1$ in the vicinity of this frequency, there are an infinite number of resonances in the intensity of diffraction radiation.

Consider now the changes in the radiation field arising for $h \neq 0$ in that case when only zeroth spatial harmonic propagates in the lower half-space without decay. Figure 6 shows the frequency dependence of the intensity of radiation in the lower half-space for the electron beam velocity $\beta=$ 0.89 . Over the whole frequency range considered here, we have $\varepsilon(k)<0$ and $\mu(k)<0$. For parameters $k_{\varepsilon}=1.0$ and $k_{\mu}=0.5$ (see Figure 6(a)), a periodic boundary within the considered frequency range and for not very large $h$ does not support propagation of surface waves, and $W_{0}^{T}(k)$ behaves almost in the same way as in the case $h=0$.

For parameters $k_{\varepsilon}=0.5$ and $k_{\mu}=1.0$ (see Figure 6(b)), the periodic boundary supports the propagation of backward surface waves (see Figure 2(a)). This fact changes significantly the $W_{0}^{T}(k)$ behavior: at $k \approx 0.4365$ a certain resonance appears due to the excitation of the surface wave with 


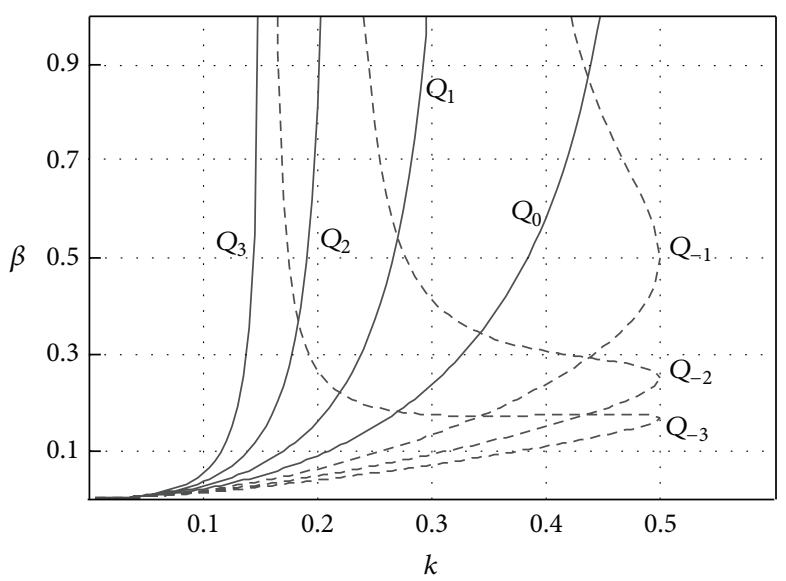

(a)

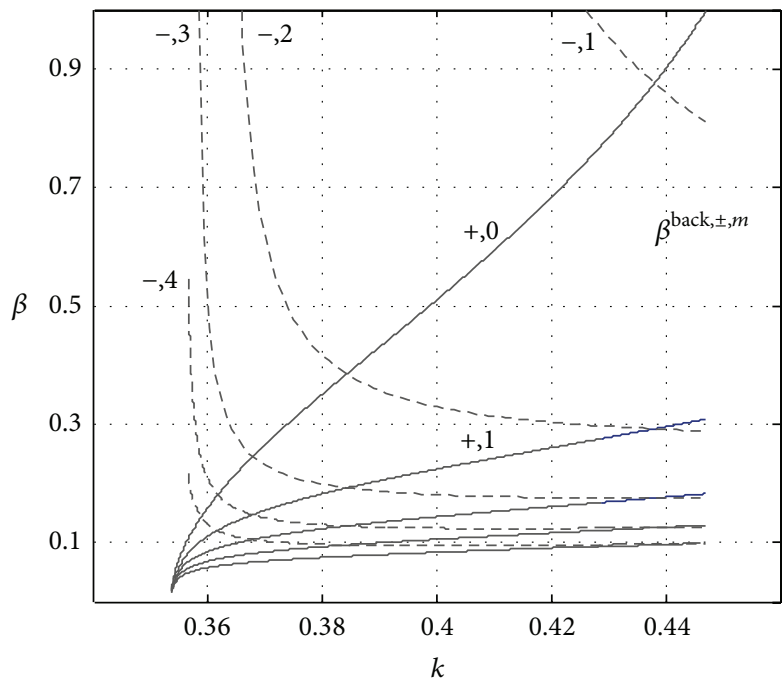

(b)

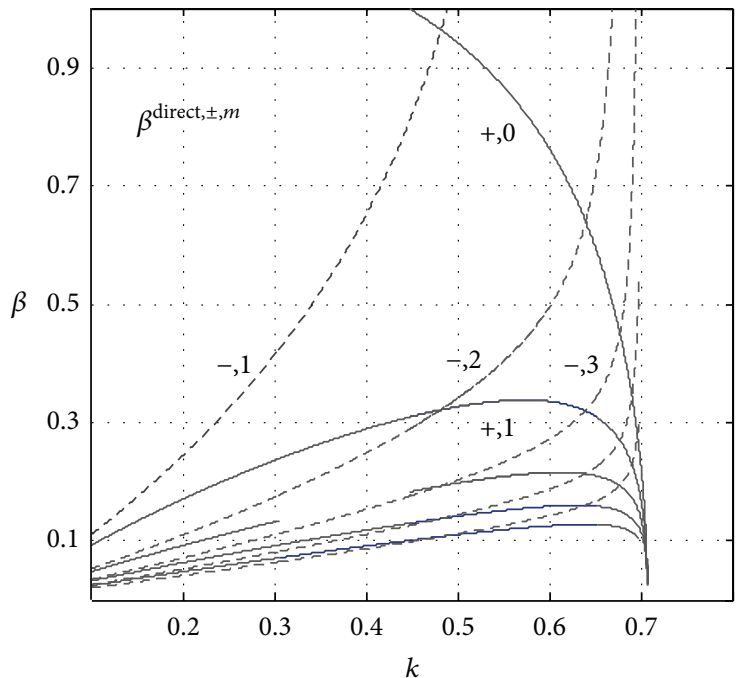

(c)

FIGURE 5: (a) Configuration of boundaries $Q_{n}$; (b) phase velocities of backward eigenwaves, $k_{\varepsilon}=0.5$ and $k_{\mu}=1.0$; (c) phase velocities of direct eigenwaves, $k_{\varepsilon}=1.0$ and $k_{\mu}=0.5$.

eigenpropagation constant $\bar{\Phi}_{0}^{\text {back,-,1 }}=-\bar{\Phi}_{0}^{\text {back }}+1=0.49$. The phase velocity of this wave is $\beta^{\text {back,-,1 }}=k / \bar{\Phi}_{0}^{\text {back,-, } 1}=0.89$ and coincides with the beam velocity $\beta$.

Resonances in reverse Vavilov-Cherenkov radiation are possible in the region of parameter values $k$ and $\beta$ such that $\beta^{2}>[\varepsilon(k) \mu(k)]^{-1}$ and $\operatorname{Re}_{n}^{\mathcal{\varepsilon}, \mu}(k, \beta)=0$ for all $n \neq 0$ (only the fundamental spatial harmonic propagates in the dispersive medium without decay). It is also necessary that in this domain (in the domain $\mathrm{VCh}_{k, \beta}$ ) the synchronism conditions $\beta=\beta^{\text {back, }, m}=k / \bar{\Phi}_{0}^{\text {back, }, m}$ for certain $m$ can be implemented.

Naturally, the selected values $k_{\varepsilon}$ and $k_{\mu}$ should ensure the existence of such a domain. For example, when $0.5<$ $k_{\varepsilon}<k_{\mu}$, the values $\beta=\beta^{\text {back, }, m}=k / \bar{\Phi}_{0}^{\text {back, }, m}$ do not fall into the domain $\mathrm{VCh}_{k, \beta}$; but for $k_{\varepsilon}=0.1$ and $k_{\mu}=0.55$ in the domain $\mathrm{VCh}_{k, \beta}$, which is marked in Figure $7(\mathrm{~b})$ with oblique hatching, the surface waves whose phase velocity coincides with the velocity of electron beam may already exist. The latter case is particularly interesting because (i) the resonances in the reverse VChR can appear for rather small values $\beta$ (Figure $7(\mathrm{c})$ ) and thus at low velocities of the particles beam and (ii) the accumulation point of the frequency spectrum $k_{\text {accum }}=k_{\varepsilon} / \sqrt{2} \approx 0.0707$ falls into the region $\mathrm{VCh}_{k, \beta}$ and within it the existence of a large number of resonances in reverse VChR (Figures 7(a) and $7(c))$ is possible. It should be pointed out once again that these resonances appeared due to the implementation of synchronism mechanism that is the coincidence of beam velocity with the phase velocity of one or another surface wave.

The data presented in Figure 8 allow us to estimate the influence of parameters of the dispersive media $k_{\varepsilon}$ and $k_{\mu}$ on the intensity of reverse VChR. The lines $W_{0}^{T}\left(k_{\varepsilon}, k_{\mu}\right)=$ const (Figure $8(\mathrm{~b})$ ) are calculated for parameters $k_{\varepsilon}$ and $k_{\mu}$ from the domain $\left[0 \leq k_{\varepsilon} \leq 0.12\right] \times\left[0.35 \leq k_{\mu} \leq 0.7\right]$ and for $k=0.08$, $\beta=0.2$, and $h=0.4$. Under such choice of parameters, the region bounded by the curves $Q_{0}$ and $Q_{-1}$ is the domain 

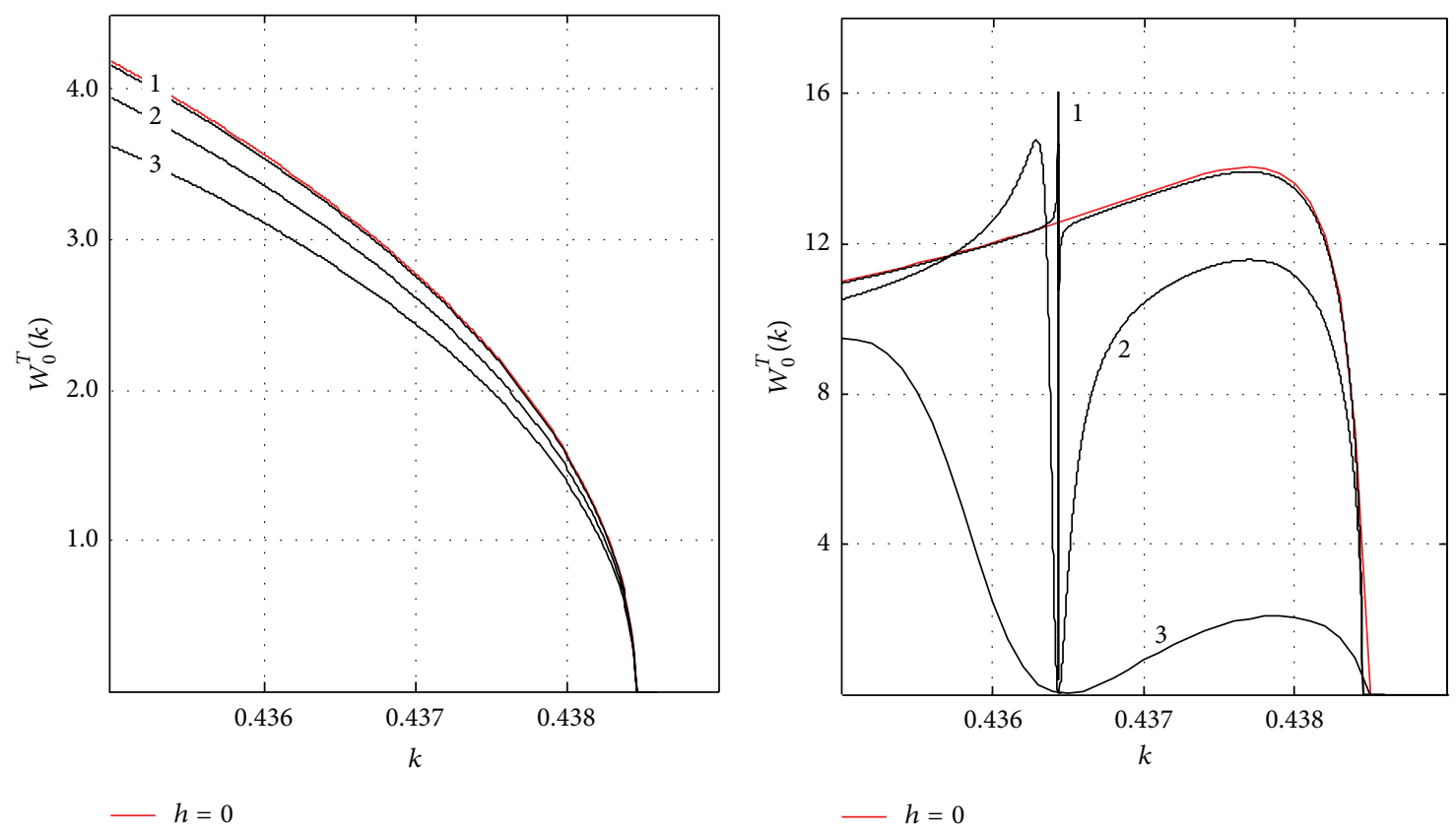
(1) $h=0.01$
(1) $h=0.01$
(2) $h=0.1$
(2) $h=0.1$
(3) $h=0.4$
(3) $h=0.4$

(a)

(b)

FIGURE 6: Change in frequency characteristics of radiation intensity with an increase in the height of corrugation mounts of periodically rough boundary.

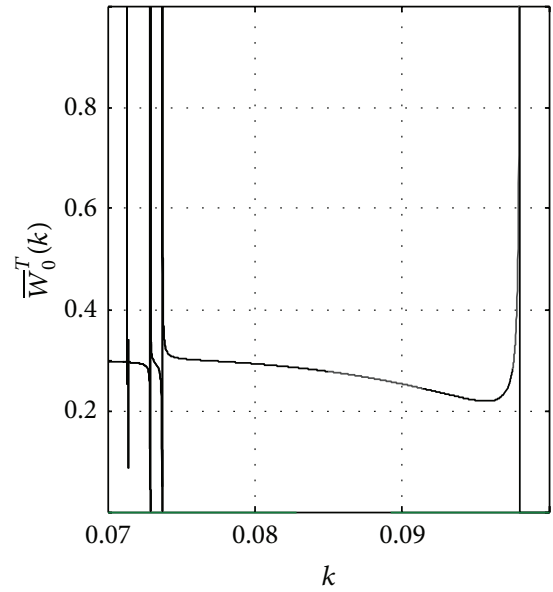

(a)

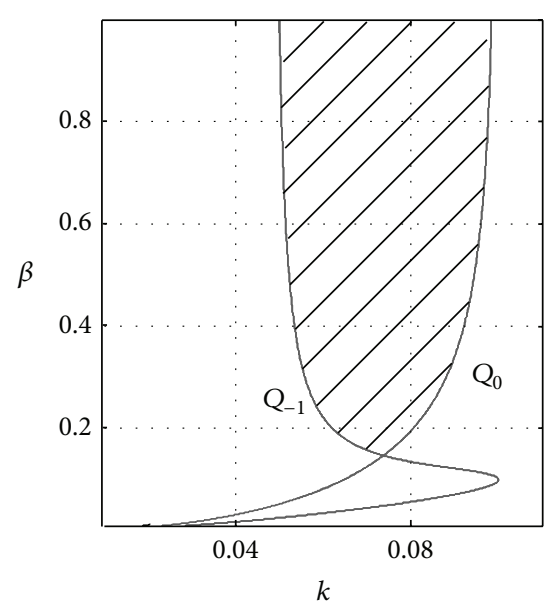

(b)

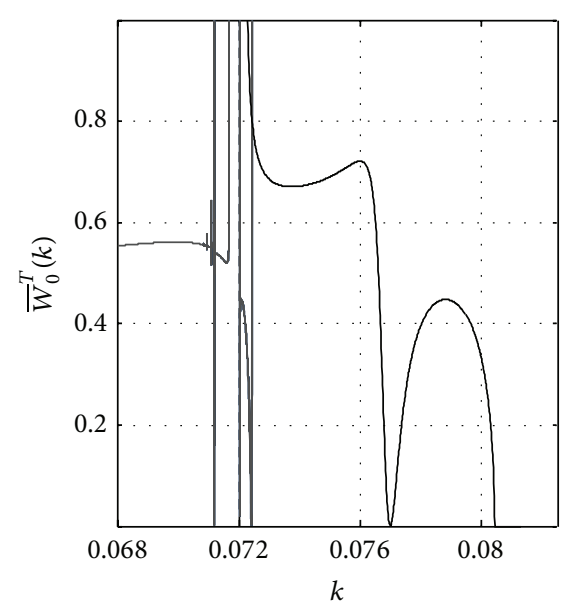

(c)

FIGURE 7: (a), (c) Dependencies $\bar{W}_{0}^{T}(k)$, characterizing the radiation intensity in the half-space $z<0$ for $\beta=0.89$ and $\beta=0.2$; (b) domain $\operatorname{VCh}_{k, \beta}$. All for $k_{\varepsilon}=0.1, k_{\mu}=0.55$, and $h=0.4$. Here and further on $\bar{W}_{0}^{T}(\cdots)=W_{0}^{T}(\cdots) / \max _{(\cdots)} W_{0}^{T}(\cdots)$.

$\mathrm{VCh}_{k_{\varepsilon}, k_{\mu}}$. The first pronounced resonant fall in the intensity of the reverse VChR (the locus of corresponding points forms the straight line in the given range of variables $k_{\varepsilon}$ and $k_{\mu}$ ) corresponds to the synchronism of electron beam with the surface wave having phase velocity $\Phi_{0}^{\text {back,-,1. }}$. Sequence of resonances is clearly displayed in Figure 8(a), where a plot
$W_{0}^{T}\left(k_{\varepsilon}\right)$ for $k_{\mu}=0.55$ is presented. The low-Q resonant fall of intensity of the reverse VChR manifests itself when $k_{\varepsilon}$ increases and then as you get closer to the value $k_{\varepsilon}=0.113$ at which the chosen frequency $k=0.08$ is an accumulation point. As we have mentioned above and in [13] in the vicinity of accumulation point, the number of high- $Q$ resonances, 


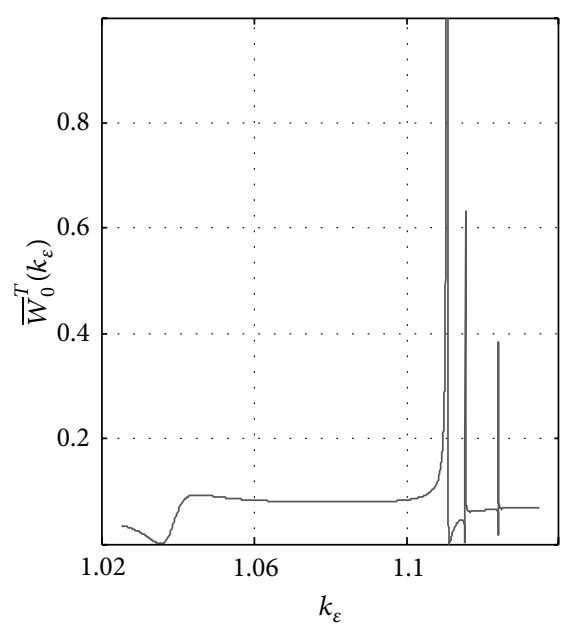

(a)

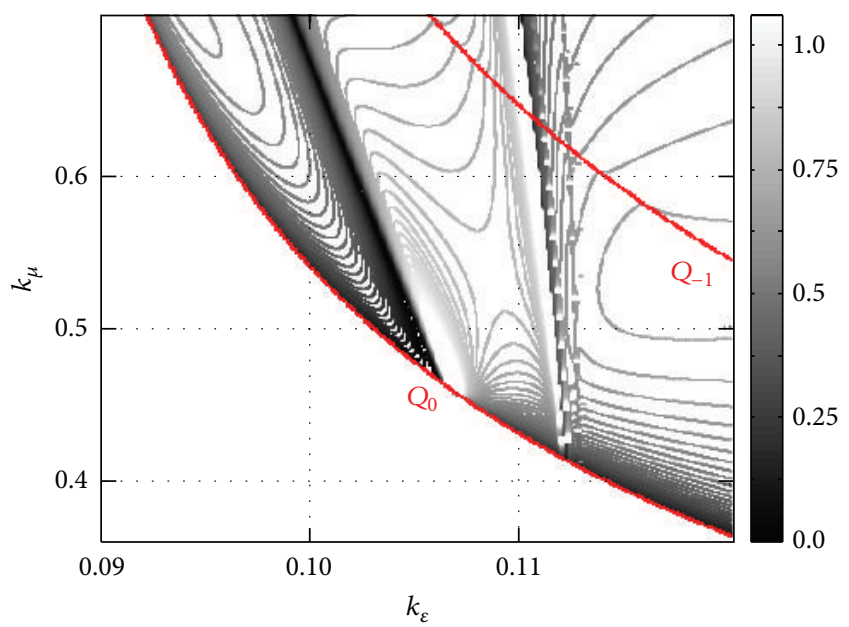

(b)

FIGURE 8: Influence of parameters $k_{\varepsilon}$ and $k_{\mu}$ onto the intensity of the radiation in the lower half-space: (a) plot $\bar{W}_{0}^{T}\left(k_{\varepsilon}\right)$ for $k_{\mu}=0.55$; (b) contour plots $\bar{W}_{0}^{T}\left(k_{\varepsilon}, k_{\mu}\right)=$ const.

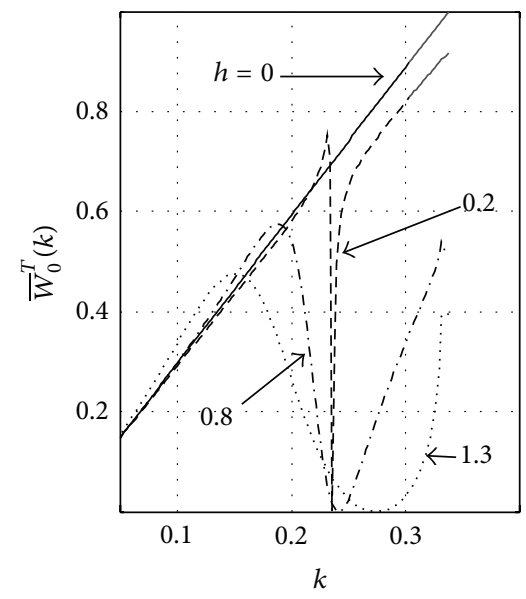

(a)

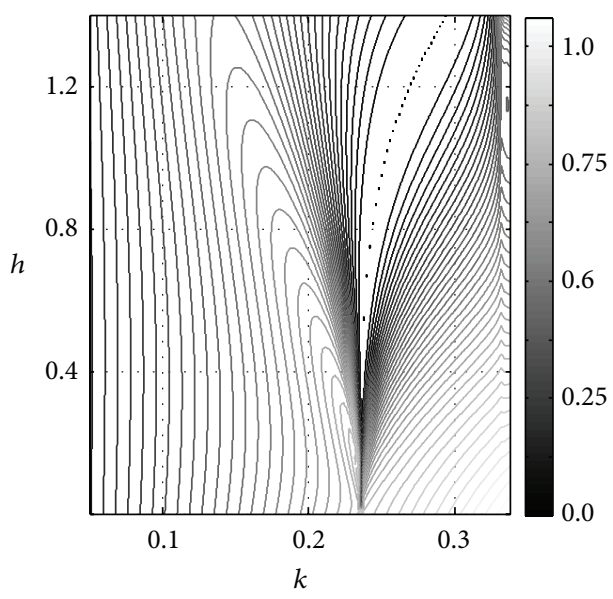

(b)

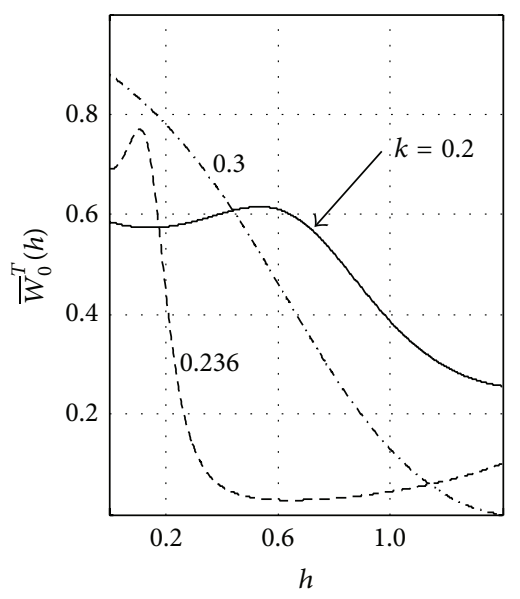

(c)

Figure 9: (a), (c) Plots $\bar{W}_{0}^{T}(k)$ for several fixed values $h$ and plots $\bar{W}_{0}^{T}(h)$ for several fixed values $k$; (b) contour plots $\bar{W}_{0}^{T}(k, h)=$ const. All for $\varepsilon=-0.84, \mu=-4.84$, and $\beta=0.89$.

causing a sharp drop and a sharp rise in $W_{0}^{T}\left(k_{\varepsilon}\right)$, increases significantly.

Influence of profiling depth $h$ onto the intensity of VChR shows itself differently depending on the values of other parameters and, in fact, depending on whether a periodic boundary between two media at such values of parameters is able to support the propagation of forward or backward surface waves and whether the synchronization of electron beam velocity with phase velocity of these waves is possible.

Consider periodic boundary of the nondispersive lefthanded medium, $\mu=-4.84, \varepsilon=-0.84$, and $\beta=0.89$, Figure 9. The resonance dip to zero of intensity of reverse VChR, which is clearly visible in Figure 9(b), is associated with the excitation of a surface wave, and the quality factor of the corresponding resonance is significantly reduced with the increase of $h$. Two other fragments of Figure 9 allow considering the important details in the behavior of the dependencies $W_{0}^{T}(k)$ (for some fixed values $h$ ) and $W_{0}^{T}(h)$ (for some fixed values $k$ ) which may be lost in the analysis of the overall picture of the contour plots $W_{0}^{T}(k, h)=$ const (Figure 9(b)).

In the case of the dispersive medium and the parameter values $k_{\varepsilon}, k_{\mu}, k$, and $\beta$, guaranteeing the existence of direct surface waves, dependencies $W_{0}^{T}(h)$ are almost monotonous. In the region of the backward surface waves, intensity of the reverse VChR varies essentially differently. For example, in the case of parameters already considered above $k_{\varepsilon}=0.1$, $k_{\mu}=0.55$, and $\beta=0.89\left(k_{\text {con }} \approx 0.0707\right)$, functions $W_{0}^{T}(h)$ have a pronounced peak due to the coincidence of 


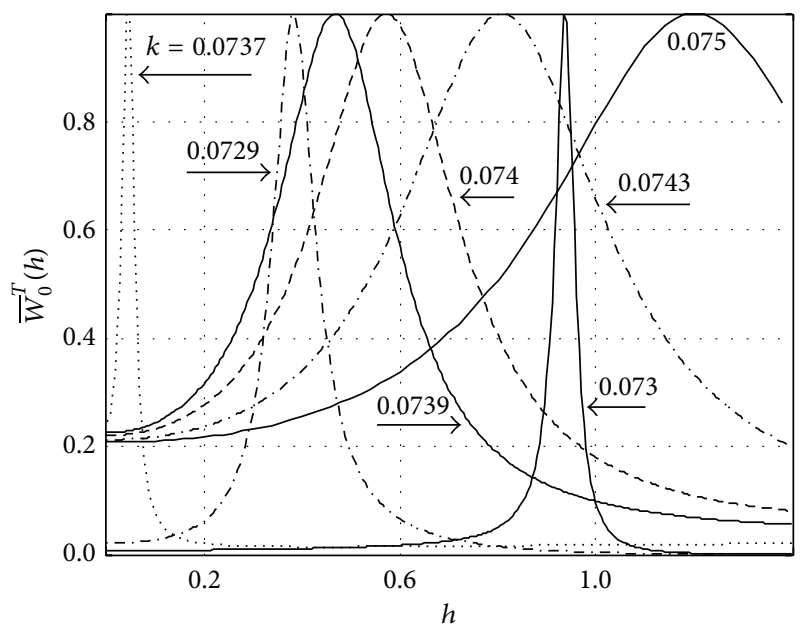

Figure 10: Curves of $\bar{W}_{0}^{T}(h)$ for several fixed values of $k$.

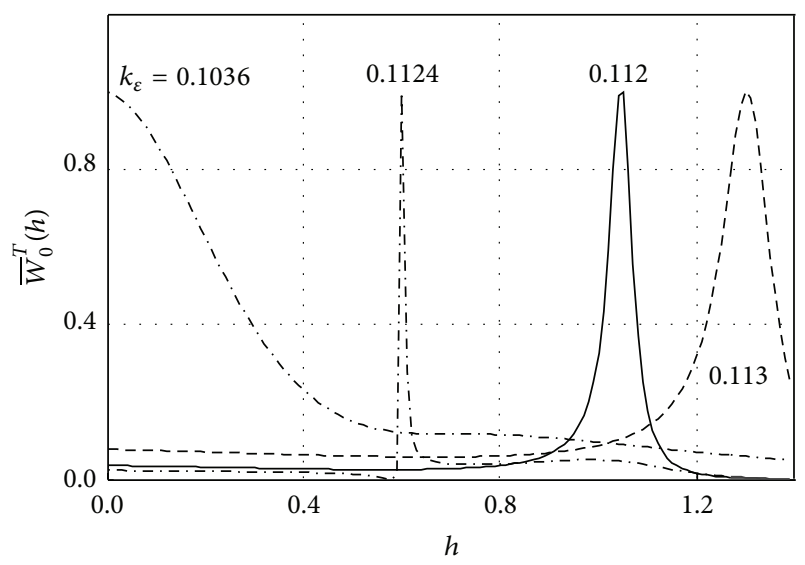

FIGURE 11: Influence of profiling depth of periodic boundary between two media onto the intensity of VChR. Plots $\bar{W}_{0}^{T}(h)$ for various $k_{\varepsilon}$.

the electrons beam velocity with phase velocity of one of the surface waves; and this maximum occurs at different values of $h$ for different values of $k$ from the frequency interval comprising $k_{\text {accum }}$ (Figure 10). Also, it is possible to characterize the behavior and features of $W_{0}^{T}(h)$ calculated for fixed $k_{\mu}=0.55, k=0.08$, and $\beta=0.2$, and for different values $k_{\varepsilon}$ (Figure 11). Location of peaks of function $W_{0}^{T}(h)$ is quite sensitive to changes in $k_{\varepsilon}$ value and this fact can be used to solve problems related to the definition of parameters of dispersive materials. The results similar to that shown in Figure 10 may be useful in the selection of depth profiling of left-handed dispersive medium capable of supporting over its surface the propagation of eigenwave of a required type.

\section{Conclusions}

The resonances in reverse VChR produced by the charged particles beam passage over periodic boundary of dispersive left-handed medium are found out and studied.
Mathematical modeling and computational experiment are the main methodological components of this work devoted to the study of a series of features in the diffraction radiation generated by a flat, density modulated electron beam, moving over periodically rough boundary between conventional media and media with frequency dependent parameters. Conditions of radiation initiation, radiation intensity, and possibility of the existence of different resonance effects in reverse VChR associated with the excitation of surface waves of the periodic boundary have been studied analytically and numerically in the approximation of a given current. The possibilities and perspectives for efficient control of the characteristics of the reverse VChR, that may be performed by changing, for example, characteristic frequencies $k_{\varepsilon}$ and $k_{\mu}$ in constitutive parameters of dispersive medium or/and the height of the corrugation mounts $h$ of the periodic surface, are found out and examined.

The numerical results discussed in the paper concern the limited number of specific situations. But the models and algorithms created for their analysis can be used for a more detailed research into the physics of diffraction radiation, for a correct formulation and efficient solution to a number of inverse problems having considerable practical interest.

\section{Conflict of Interests}

The authors declare that there is no conflict of interests regarding the publication of this paper.

\section{Acknowledgment}

This work has been carried out under financial support of the Ministry of Education and Science of Kazakhstan.

\section{References}

[1] P. A. Cherenkov, "Visible radiation produced by electrons moving in a medium with velocities exceeding that of light," Physical Review, vol. 52, p. 378, 1937.

[2] I. Y. Tamm and I. M. Frank, "Coherent visible radiation of fast electrons passing through matter," Comptes Rendus de l'Academie Bulgare des Sciences, vol. 14, p. 109, 1937.

[3] J. V. Jelley, Cherenkov Radiation and Its Applications, Pergamon Press, London, UK, 1958.

[4] V. P. Shestopalov, The Smith-Purcell Effect, Nova Science Publishes, New York, NY, USA, 1997.

[5] Y. O. Averkov and V. M. Yakovenko, "Cherenkov radiation by an electron bunch that moves in a vacuum above a left-handed material," Physical Review B-Condensed Matter and Materials Physics, vol. 72, no. 20, Article ID 205110, 2005.

[6] Z. Y. Duan, B.-I. Wu, S. Xi, H. S. Chen, and M. Chen, "Research progress in reversed cherenkov radiation in double-negative metamaterials," Progress in Electromagnetics Research, vol. 90, pp. 75-87, 2009.

[7] S. Xi, H. Chen, T. Jiang et al., "Experimental verification of reversed cherenkov radiation in Left-Handed metamaterial," Physical Review Letters, vol. 103, no. 19, Article ID 194801, 2009.

[8] Y. K. Sirenko and S. Strom, Eds., Modern Theory of Gratings. Resonant Scattering: Analysis Techniques and Phenomena, Springer, New York, NY, USA, 2010. 
[9] V. P. Shestopalov, Y. A. Tuchkin, A. Y. Poyedinchuk, and Y. K. Sirenko, New Solution Methods for Direct and Inverse Problems of the Diffraction Theory. Analytical Regularization of the Boundary Value Problems in Electromagnetic Theory, Osnova, Kharkov, Ukraine, 1997 (Russian).

[10] P. Melezhik, A. Poyedinchuk, N. Yashina, and G. Granet, "Periodic boundaries of metamaterials: eigen regimes and resonant radiation," Journal of Optics A: Pure and Applied Optics, vol. 9, no. 9, pp. S403-S409, 2007.

[11] V. P. Shestopalov, A. A. Kirilenko, S. A. Masalov, and Y. K. Sirenko, Resonance Wave Scattering. Diffraction Gratings, Naukova Dumka, Kiev, Ukraine, 1986, (Russian).

[12] Y. K. Sirenko and L. G. Velychko, "The features of resonant scattering of plane inhomogeneous waves by gratings: model problem for relativistic diffraction electronics," Telecommunications and Radio Engineering, vol. 55, p. 33, 2001.

[13] P. Melezhik, A. Poyedinchuk, N. Yashina, G. Granet, and M. Ney, "Radiation from surface with periodic boundary of metamaterials excited by a current," Progress in Electromagnetics Research, vol. 65, pp. 1-14, 2006. 

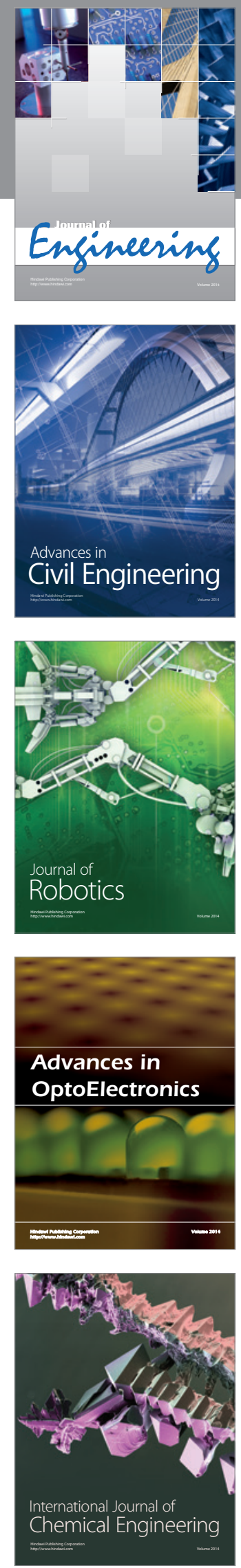

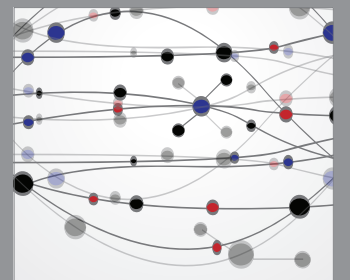

The Scientific World Journal
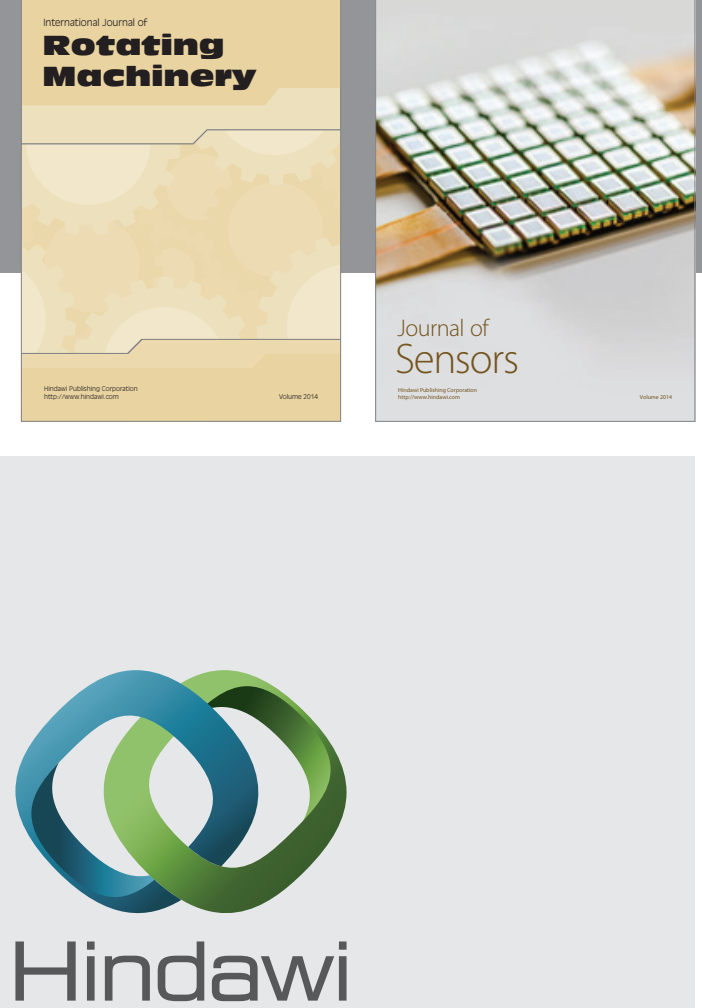

Submit your manuscripts at http://www.hindawi.com
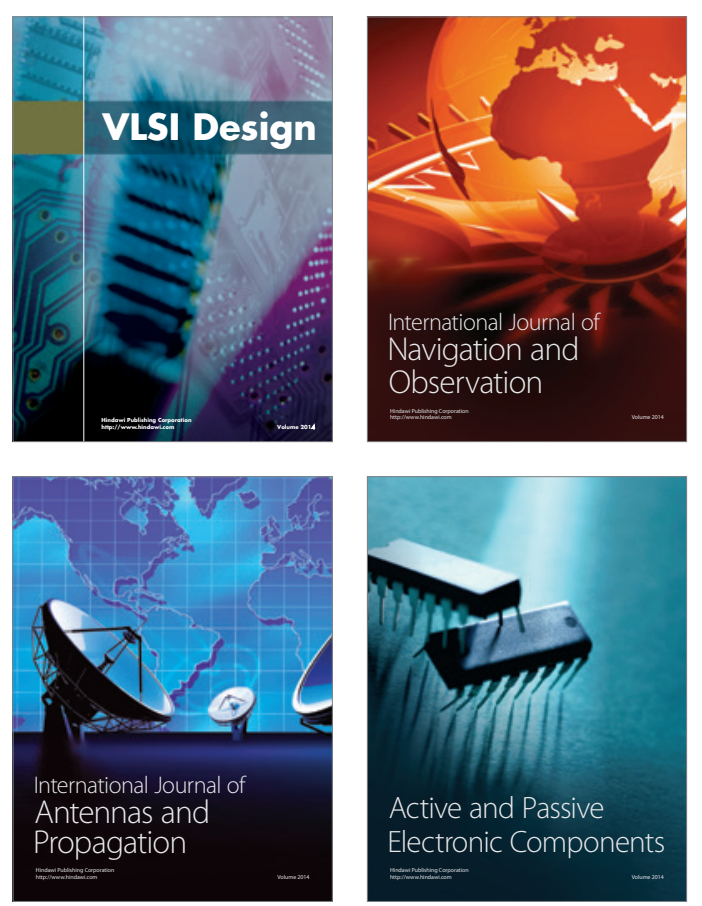
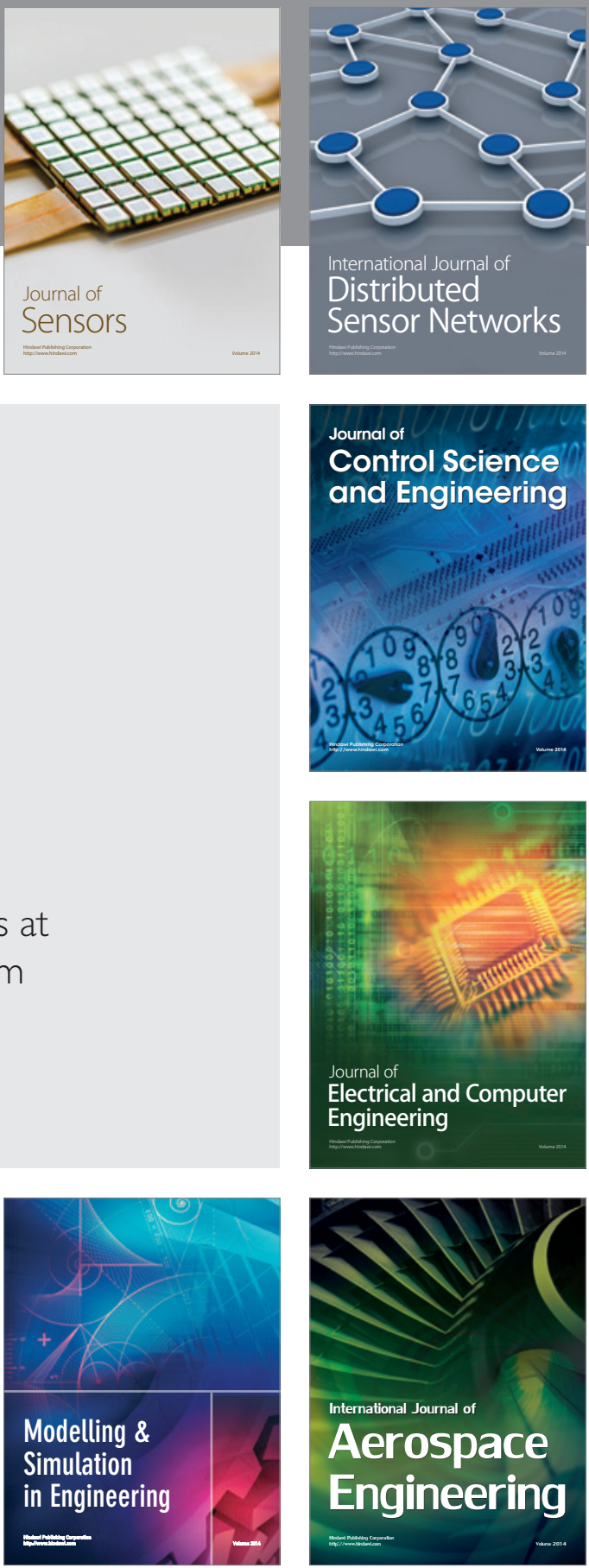

Journal of

Control Science

and Engineering
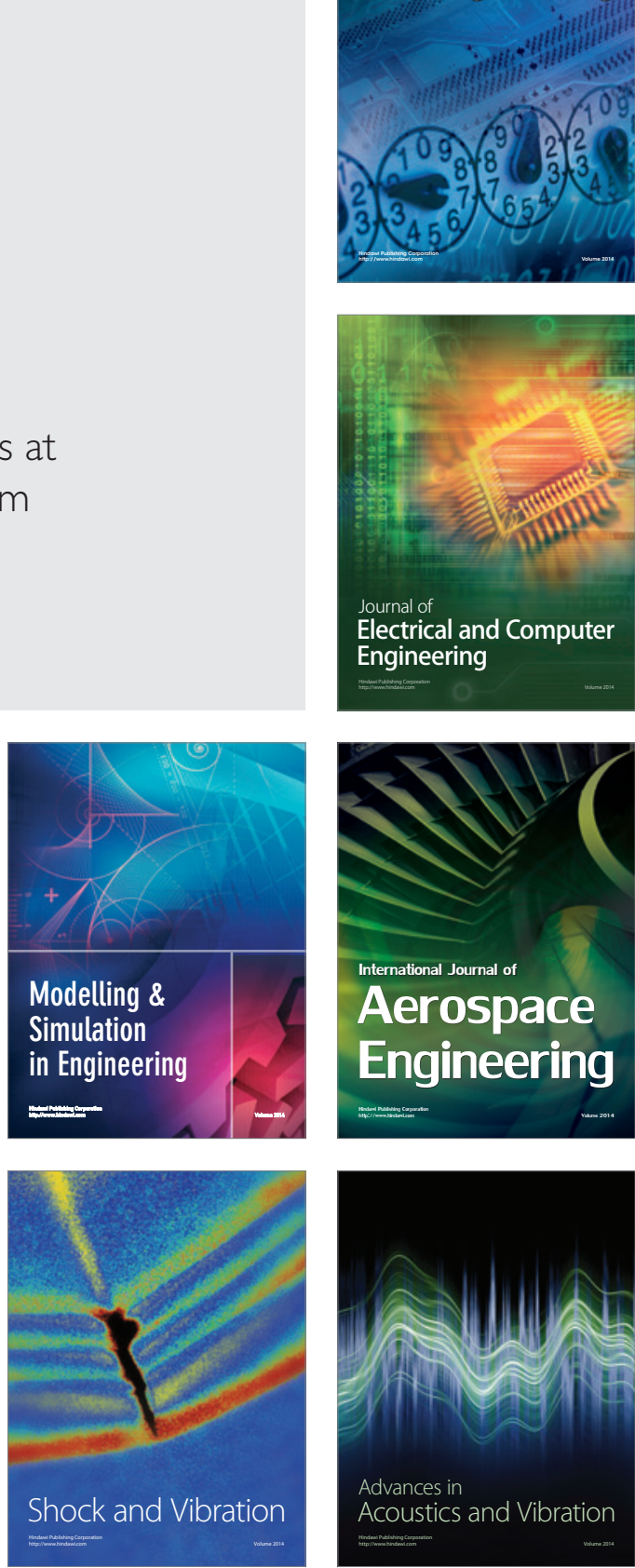